\title{
Therapeutic Evaluation of Artesunate in IgA Nephropathy (TEATING) Study: A study protocol of a multicenter, double-blind, randomized, placebo- controlled trial
}

Qi Chen ( $\nabla$ chenqi_smq@126.com)

Beijing University of Chinese Medicine Affiliated Dongzhimen Hospital

\section{Zi Wang}

Peking University First Hospital

Jicheng Lv

Peking University First Hospital

Lijun Liu

Peking University First Hospital

Hang Li

Peking Union Medical College Hospital

\section{Weiwei Sun}

Beijing University of Chinese Medicine Affiliated Dongzhimen Hospital

\section{Yanhong Huo}

7th medical center of PLA hosipal

\section{Yingbo Guo}

Dongfang Hospital

\section{Cun Shen}

Beijing Hospital of Traditional Chinese Medicine

\section{Shichao Li}

Beijing University of Chinese Medicine Affiliated Dongzhimen Hospital

\section{Zhenjie Chen}

Beijing University of Chinese Medicine Affiliated Dongzhimen Hospital Jingwei Zhou

Beijing University of Chinese Medicine Affiliated Dongzhimen Hospital

\section{Research Article}

Keywords: IgA nephropathy, artesunate, proteinuria

Posted Date: May 3rd, 2021 
DOl: https://doi.org/10.21203/rs.3.rs-324565/v1

License: (c) (1) This work is licensed under a Creative Commons Attribution 4.0 International License. Read Full License 


\section{Abstract \\ Background}

IgA nephropathy is the most common glomerular disease and a common cause of progression to endstage renal disease in patients with kidney diseases. Proteinuria levels are critical to the prognosis of patients with IgA nephropathy, but many patients are still unable to effectively control proteinuria levels after receiving adequate RAAS blockers. Antimalarial drugs have shown good efficacy in the treatment of kidney disease in previous studies; however, there have been no strict designed randomized controlled trials to confirm the clinical efficacy of artesunate in IgA nephropathy patients. Therefore, we designed this clinical trial to compare the effect of artesunate versus placebo in patients with IgA nephropathy.

\section{Methods}

This study was a randomized, double-blind, three-group-parallel, placebo-controlled clinical trial. One hundred and twenty eligible IgA nephropathy patients at risk of progression were randomly divided into artesunate $100 \mathrm{mg}$ group, artesunate $50 \mathrm{mg}$ group, and placebo group. Changes in proteinuria and renal function were measured after 6 months of intervention. The levels of Gd-lgA1, Anti-Gd-lgA1 in the patient's blood will also be tested to further understand possible immune mechanisms.

\section{Discussion}

Clinical evidence for artesunate treatment of IgA nephropathy is currently lacking, and we expect that the results of this trial will provide high-quality clinical evidence for artesunate as a treatment option for IgA nephropathy in the future.

\section{Trial registration:}

Chinese Clinical Trial Registry: ChiCTR2000038104, registration date: 10 September 2020. http://www.chictr.org.cn/edit.aspx?pid=61338\&htm=4.

\section{Background}

Immunoglobulin A $(\lg A)$ nephropathy, characterized by prominent mesangial $\lg A$ deposition that is obfuscated on an immunofluorescence microscope, is the most common glomerular disease in the world [1]. The natural history of IgA nephropathy was highly heterogeneous, although clinical data showed that $50 \%$ of IgA nephropathy patients experienced sustained clinical remission, up to $40 \%$ of patients still developed end-stage renal disease (ESRD) within 20 years, and up to $40 \%$ experienced a persistent decline in renal function [2-4]. Large amounts of proteinuria are considered a major risk factor for IgA nephropathy progression, and current KDIGO Clinical Practice Guideline recommended that patients with 
IgA nephropathy with persistent proteinuria greater than $1 \mathrm{~g} /$ day be treated with corticosteroid therapy for 6 months with adequate supportive care (maximum tolerated renin-angiotensin-aldosterone system inhibitor therapy and blood pressure control) [5]. However, two recent large clinical trials of corticosteroids therapy for IgA nephropathy have reported significant safety concerns with systemic

immunosuppressants [6, 7], which has forced us to re-examine current treatment strategies. Although the latest rituximab, eculizumab, targeted-release budesonide may be a potential treatment for IgA nephropathy, it is still far from clinical practice because of the high cost and unclear risks of adverse events [8-10]. In the absence of more effective and available control therapies for IgA nephropathy with a high risk of progression, more easier and safer supportive therapies are warranted.

Antimalarial drugs provide a new treatment approach for the management of IgA nephropathy. In a recent randomized controlled study, IgA nephropathy patients with RAAS blockers received hydroxychloroquine (HCQ) for six months and had a nearly $50 \%$ reduction in proteinuria, which had a significant difference from those patients who received RAAS blockers without HCQ (a 10\% increase) [11]. HCQ also has been reported in clinical studies to reduce Systemic Lupus Erythematosus disease activity index (SLE-DAI) and mortality in lupus nephritis and is becoming the standard treatment for lupus nephritis [12]. Importantly, these studies also identified fewer side effects with HCQ than with corticosteroid, which gives us more confidence that antimalarial drugs may be an effective management measure for IgA nephropathy.

Artesunate (ART) is a derivative of artemisinin (ARS), an effective component extracted from traditional Chinese herb Artemisia annua $L$, with improved pharmacological properties. ART has similar immunomodulating effects to other antimalarial drugs, such as hydroxychloroquine (HCQ) and chloroquine (CQ), but has fewer side effects, especially without the visual loss caused by HCQ. Many experimental pieces of evidence have shown that ARS and its derivatives (ARSs) have good antiinflammatory and immunomodulatory functions in kidney disease, especially its unique role in regulating the glomerular barrier [13]. Although a large amount of experimental evidence has been accumulated, there is still a lack of randomized controlled clinical trials to verify the potential effect of ART in the treatment of IgA nephropathy. This multicenter, double-blind, randomized, placebo-controlled study was designed to estimate the efficacy and safety of ART in IgA nephropathy. We present the protocol in accordance with the SPIRIT reporting checklist.

\section{Methods And Design}

\section{Study design}

This study was designed as a double-blind, randomized, three-group-parallel, placebo-controlled clinical trial. One hundred and twenty patients with IgA nephropathy will be recruited, and the overall study design will be divided into two phases. In the pre-randomization phase, all participants will be confirmed to have received at least 3 months of RAAS blockers therapy and to have blood pressure within 130/80 $\mathrm{mmHg}$ before entering the next phase. After qualification confirmation and informed consent, the enrolled patients entered the post-randomization intervention phase. All participants were randomly divided into 
ART 100mg group, ART 50mg group, and placebo group, for 6 months of intervention. The flow diagram of the study progress is shown in Fig. 1. Each participant will be assigned eight visits, including 3 months before the randomization (visit 1), 2 months before the randomization (visit 2), 1 month before the randomization (visit 3), randomization (visit 4), 1 month after the intervention (Visit 5), 2 months after the intervention (visit 6), 3 months after the intervention (visit 7), and 6 months after the intervention (Visit 8). The schedule for enrollment, intervention, and assessment is shown in Table 1. The primary outcome measures will be determined by the change in proteinuria in the three groups after 6 months of intervention. The secondary outcome measures will be the changes in urinary protein creatinine ratio and estimated glomerular filtration rate (eGFR) from baseline after 1,3凶6 months of intervention, and the change in serum galactose-deficient IgA1 (Gd-IgA1) and Anti- Gd-IgA1 levels in patients will also be observed. We will stratify progression risk in IgA patients and retrospectively evaluate the effect of ART on proteinuria levels in IgA patients with different progression risks. The safety assessment of ART will be carried out throughout the study. The study protocol has been approved by the Ethics Committee of Dongzhimen Hospital Affiliated to Beijing University of Chinese Medicine (Number: DZMEC-KY-2020-30) and registered in the Chinese Clinical Trial Registry (ChiCTR) (Number: ChiCTR2000038104).

Table 1

Schedule of recruitment, interventions, and assessments.

a Items include: Urea, Creatinine, Total bilirubin, Serum glutamic pyruvic transaminase, Serum glutamic-oxaloacetic transaminase, Alkaline phosphatase, Sodium, Potassium, Calcium, Phosphorus, Total protein, Albumin, Uric acid.

${ }^{\mathrm{b}}$ Items include: Urea, Creatinine, Sodium, Potassium.

${ }^{c}$ Items include: Gd-IgA1, Anti-Gd-IgA1(IgG/lgA).

Abbreviation: HCG, human chorionic gonadotropin.

This protocol is reported according to the Standard Protocol Items: Recommendations for Interventional Trials (SPIRIT) 2013 statement [14]. The SPIRIT checklist is listed in Supplement Table 1.

\section{Participant recruitment}

Inclusion criteria

Inclusion criteria for this study include:1) Ages 18-75; 2) Primary IgA nephropathy confirmed by renal biopsy; 3 ) Persistent proteinuria $\geq 1.0 \mathrm{~g} /$ day after receiving adequate RAAS blockers treatment (proteinuria $\geq 1.0 \mathrm{~g} /$ day both in visit 1 and visit 3 ); 4) eGFR $\geq 30 \mathrm{ml} / \mathrm{min}$ per $1.73 \mathrm{~m}^{2}$ calculated by Chronic Kidney Disease Epidemiology Collaboration (CKD- EPI) equation.

Exclusion criteria

Exclusion criteria included: Patients who had to be treated with corticosteroid immunotherapy (microplastic nephropathy with IgA deposition and the presence of crescents in more than $50 \%$ of the 
glomeruli of renal biopsy within 12 months); Patients who must be treated with other immunosuppressants (such as calcineurin inhibitors, cyclophosphamide or mycophenolate mofetil); Patients receiving systemic immunosuppressive therapy in the past 6 months; Patients with uncontrolled hypertension(systolic blood pressure $>160 \mathrm{mmHg}$ or diastolic blood pressure $>110 \mathrm{mmHg}$ ); Secondary IgA nephropathy; Acute kidney injury; Malignant tumors; Patients with serious diseases of other systems (such as liver damage, heart damage, diseases of the blood system, systemic infections, digestive ulcers); Women who are planning or having pregnant; Patients with cognitive impairment who cannot sign the informed consent.

Withdrawal criteria

The subjects who no longer meet the inclusion criteria, have poor compliance, need immunosuppressive therapy, or have any other reason for the violation of the protocol will be determined by the investigators. These patients will be required to continue regular study visits as scheduled in the protocol after discontinuation of treatment.

Dropout criteria

Patients who refuse to participate in the study visits for personal reasons, or the researchers were unable to contact through all available means will be regarded as a dropout.

Termination criteria

Patients had a severe liver or kidney damage during the trial (ALT or AST exceeding twice the normal upper limit, acute kidney injury); Severe clinical discomfort caused by the drug (such as severe nausea and vomiting, severe dizziness, and headache); Severe drug allergies. All criteria and decisions will be jointly determined by two or more physicians with the title of deputy chief or higher

\section{Randomization and allocation}

Eligible patients will be randomly divided into ART $100 \mathrm{mg}$ group, ART $50 \mathrm{mg}$ group, or placebo group at a ratio of 1:1:1. Random Numbers will be generated and sorted by independent statisticians using the SAS 9.4 (SAS Institute Inc), and then patients will be divided into three groups based on the remainder of the number divided by 3 . The randomized numbers, rank order, and grouping will be stored in opaque envelopes with serial numbers, which will be kept by independent designers and statisticians throughout the study. The envelopes and study drug will be provided to enrolled patients separately by independent blind researchers.

\section{Blinding}

In this study, a double-blind approach will be applied to patients and physicians. ART and placebo were placed in packaged capsules and distributed to patients by independent researchers. At the end of the study, professional independent statisticians unblind for the first time and performed a preliminary analysis of three sets of outcome data. The designer will then perform a second unblinding to confirm the 
group and the effect. If serious adverse events occur during the study period, patients will be treated with emergency blinding and relevant treatment according to the situation.

\section{Interventions}

ART 100mg group oral 50mg ART tablets twice a day, ART 50mg group oral 25mg ART tablets twice a day, and placebo group oral equivalent placebo twice a day. ART tablets and placebo are provided by Guilin Nanyao Co. LTD. All patients will receive standard support treatment for $\lg A$ nephropathy including adequate RAAS blockers and blood pressure control. Permissible combination antihypertensive medications include diuretics, calcium channel blockers, and $\beta$-receptor blockers. Hydrochlorothiazide or loop diuretics could be used when the antihypertensive targets are difficult to achieve. Statins and aspirins are also allowed when necessary. Prohibited drugs include proprietary Chinese drugs that may reduce proteinuria (such as Tripterygium wilfordii, Penicillium notatum, Huangkui capsules, Nephritis Rehabilitation tablets, Haikun Shenxi capsules), corticosteroids, and any immunosuppressants (such as mycophenolate mofetil, cyclophosphamide, azathioprine, HCQ).

\section{Outcome measurement}

The primary outcome was defined as the change in 24-hour urinary protein quantity and percentage change of proteinuria in all three groups after 6 months of intervention. Secondary outcomes included the changes in urinary protein creatinine ratio (UPCR), eGFR, and serum Gb-IgA levels in all three groups from baseline. The clinical characteristics of the patients were collected at baseline before enrollment, including age, gender, age, course of the disease, combined disease, medication history, and so on. Throughout the follow-up, patients will be asked to take urine and blood samples, which will be performed independently at each center.

\section{Safety assessment}

The safety assessment includes any serious adverse event and a general adverse event according to the WHO acute and subacute side effects of the performance and indexing criteria for assessment. Patients' vital signs and any clinical discomfort will be monitored throughout the study period.

\section{Data collection and management}

The entire data collection and preliminary evaluation of the results were done by an independent third party. The data is collected in the form of a case report form (CRF), and then reviewed and stored by the lead researcher in each center. Two researchers will independently convert the original data into electronic storage data to ensure the accuracy of the data. All relevant documentation will be retained so that safety and key efficacy outcomes can be retrospectively evaluated in the future.

\section{Sample size}

This is a double-blind, randomized, three-group-parallel, placebo-controlled clinical study, and sample size estimates were based on the change in proteinuria levels. According to the same kind of drugs (HCQ) in the treatment of IgA nephropathy study results, the average baseline proteinuria was $2.0 \pm 0.8 \mathrm{~g} / \mathrm{day}$, and 
36 participants of each arm will provide $90 \%$ of the detection ability, and assuming that the placebo group level of proteinuria has not changed, and ART treatment detect a $25 \%$ reduction in proteinuria (ART group: $0.5 \pm 0.5 \mathrm{~g} / \mathrm{d}$, the control group $0.2 \pm 0.2 \mathrm{~g} / \mathrm{d}$ ). Assuming a $10 \%$ dropout rate with a type I error rate of 0.05 , we plan to enroll 120 participants ( 40 in each group) in the study.

\section{Statistical analysis}

All results were analyzed based on the intention-to-treat (ITT) analysis. Normally distributed data are expressed as mean \pm standard deviation (SD), while non-normally distributed data are expressed as the median of the interquartile interval (IQR). The groups were compared using an independent sample T-test (for a continuous variable with a normal distribution), Wilcoxon rank-sum test (for a continuous variable with non-normal distribution), or chi-square test (for a nominal variable). The missing value is filled up by the last-observation-carried-forward method. For the initial analysis, we will not adjust for confounders or stratification, and for the secondary analysis, we will adjust for confounders for subgroup analyses, such as baseline proteinuria ( $3 \mathrm{~g} /$ day), renal function (eGFR $45 \mathrm{~m} 1 / \mathrm{min}$ per $1.73 \mathrm{~m}^{2}$ ), blood pressure level (systolic blood pressure $140 \mathrm{mmHg}$ ), MEST-C score, and the new international risk-prediction score. SAS 9.4 (SAS Institute Inc) will be used as statistical analysis tool. A 2-side $\mathrm{P} \otimes 0.05$ was considered statistically significant.

\section{Discussion}

More and more attention has been paid to the application of antimalarial drugs in kidney damage. Given that HCQ has been reported to have a good anti-proteinuria effect in IgA nephropathy patients, and ART has better pharmacological properties than HCQ, it is reasonable to believe that ART has the potential to treat IgA nephropathy. Therefore, we designed this double-blind, randomized, three-group-parallel, placebo-controlled clinical trial to evaluate the efficacy and safety of oral ART versus placebo in patients with IgA nephropathy characterized by high risk of progression in the context of conventional adequate RAAS blockers therapy.

Up to date, there have been few clinical trials in China on ARSs in the treatment of renal diseases, and only two observational clinical studies on lupus nephritis have reported that ARSs can significantly reduce proteinuria and regulate immune function in patients with lupus nephritis[15, 16]. Studies on the treatment of IgA nephropathy with ART were mainly based on animal models and cell experiments, and a large amount of laboratory evidence indicated that the improvement effect of ART on IgA nephropathy may be based on its strong anti-inflammatory and immune-regulatory properties, as well as its ability to regulate oxidative stress [13]. An animal experiment found that the combination of ARS and HCQ can improve the barrier function of the glomerular filtration membrane and regulate the immune response in IgA nephropathy rats[17]. One team demonstrated that dihydroartemisinin (the main active metabolite of ART) can attenuate the proliferation of human mesangial cells induced by aggregated IgA1 through the mTOR signaling pathway in vitro[18]. Although there has been a lot of current laboratory evidence that ART has a good regulatory effect on the IgA nephropathy, there is still no strict randomized, placebo- 
controlled clinical trials to provide evidence of ART in the treatment of IgA nephropathy, which has largely limited the clinical use of ART in IgA nephropathy.

Our study benefits from our more sample size and the more strict design of multicenter, three-group parallel, and placebo control, and we will observe ART on the serum Gd - IgA levels in patients with IgA nephropathy, which will help us better understand the proteinuria reduction effect of ART and its possible mechanism. The defect of this study is that it is a proof-of-concept study and only followed up for 6 months, which may not initially provide clear answers to more mechanism questions. If this study achieves the expected results at the end of the trial, further clinical studies with longer follow-up periods will be conducted to confirm whether ART could be an alternative treatment option for IgA nephropathy in the future.

\section{Declarations}

\section{Ethics approval and consent to participate}

The study protocol has been approved by the Ethics Committee of Dongzhimen Hospital Affiliated to Beijing University of Chinese Medicine (Number: DZMEC-KY-2020-30).

\section{Consent for publication}

Not applicable.

\section{Availability of data and materials}

The datasets used and/or analyzed during the current study are available from the corresponding author on reasonable request and appropriate human subject approval.

\section{Competing interests}

All authors have no conflicts of interest to declare.

\section{Funding}

This work was supported by the Key Project of Beijing University of Chinese Medicine (2020-JYB-ZDGG118).

\section{Authors' contributions}

(I) Conception and design: Z. Chen, J. Zhou, and J. Lv; (II) Administrative support: W. Sun and S. Li; (III) Provision of study materials or patients: L. Liu, H. LI, Y. Hou, Y. Guo and C. Shen; (IV) Collection and assembly of data: Q. Chen, and Z. Wang; (V) Data analysis and interpretation: W. Sun and S. Li; (VI) Manuscript writing: All authors; (VII) Final approval of manuscript: All authors.

\section{Acknowledgements}


The authors would like to thank all of the research staff at all of the participating clinical sites, all of the participating providers, the organizational leaders and the funding from Beijing University of Chinese Medicine.

\section{Abbreviations}

IgAXImmunoglobulin A; ESRD: end-stage renal disease; HCQ: hydroxychloroquine; ART: artesunate; ARS: artemisinin; CQ: chloroquine; SPIRIT: the Standard Protocol Items: Recommendations for Interventional Trials; UPCR: urinary protein creatinine ratio; ITT: intention-to-treat; SD: standard deviation; IQR: interquartile interval.

\section{References}

1. D'Amico G. The commonest glomerulonephritis in the world: IgA nephropathy. Q J Med. 1987;64(245):709-27.

2. Manno C, Strippoli GF, D'Altri C, Torres D, Rossini M, Schena FP. A novel simpler histological classification for renal survival in IgA nephropathy: a retrospective study. Am J Kidney Dis. 2007;49(6):763-75. doi:10.1053/j.ajkd.2007.03.013.

3. Nolin L, Courteau M. Management of IgA nephropathy: evidence-based recommendations. Kidney Int Suppl. 1999;70:S56-62. doi:10.1046/j.1523-1755.1999.07008.x.

4. Rekola S, Bergstrand A, Bucht H. Deterioration of GFR in IgA nephropathy as measured by 51Cr-EDTA clearance. Kidney Int. 1991;40(6):1050-4. doi:10.1038/ki.1991.313.

5. KDIGO. KDIGO Clinical Practice Guideline for Glomerulonephritis. Kidney International. 2012;2:139274.

6. Lv J, Zhang H, Wong MG, Jardine MJ, Hladunewich M, Jha $V$ et al. Effect of Oral Methylprednisolone on Clinical Outcomes in Patients With IgA Nephropathy: The TESTING Randomized Clinical Trial. Jama. 2017;318(5):432-42. doi:10.1001/jama.2017.9362.

7. Rauen T, Eitner F, Fitzner C, Sommerer C, Zeier M, Otte B et al. Intensive Supportive Care plus Immunosuppression in IgA Nephropathy. N Engl J Med. 2015;373(23):2225-36. doi:10.1056/NEJMoa1415463.

8. Fellström BC, Barratt J, Cook H, Coppo R, Feehally J, de Fijter JW et al. Targeted-release budesonide versus placebo in patients with IgA nephropathy (NEFIGAN): a double-blind, randomised, placebocontrolled phase $2 b$ trial. Lancet. 2017;389(10084):2117-27. doi:10.1016/s0140-6736(17)30550-0.

9. Rosenblad T, Rebetz J, Johansson M, Békássy Z, Sartz L, Karpman D. Eculizumab treatment for rescue of renal function in IgA nephropathy. Pediatr Nephrol. 2014;29(11):2225-8. doi:10.1007/s00467-014-2863-y.

10. Lafayette RA, Canetta PA, Rovin BH, Appel GB, Novak J, Nath KA et al. A Randomized, Controlled Trial of Rituximab in IgA Nephropathy with Proteinuria and Renal Dysfunction. J Am Soc Nephrol. 2017;28(4):1306-13. doi:10.1681/asn.2016060640. 
11. Liu LJ, Yang YZ, Shi SF, Bao YF, Yang C, Zhu SN et al. Effects of Hydroxychloroquine on Proteinuria in IgA Nephropathy: A Randomized Controlled Trial. Am J Kidney Dis. 2019;74(1):15-22. doi:10.1053/j.ajkd.2019.01.026.

12. Ponticelli C, Moroni G. Hydroxychloroquine in systemic lupus erythematosus (SLE). Expert Opin Drug Saf. 2017;16(3):411-9. doi:10.1080/14740338.2017.1269168.

13. Xia M, Liu D, Liu Y, Liu H. The Therapeutic Effect of Artemisinin and Its Derivatives in Kidney Disease. Front Pharmacol. 2020;11:380. doi:10.3389/fphar.2020.00380.

14. Chan AW, Tetzlaff JM, Altman DG, Laupacis A, Gøtzsche PC, Krleža-Jerić K et al. SPIRIT 2013 statement: defining standard protocol items for clinical trials. Ann Intern Med. 2013;158(3):200-7. doi:10.7326/0003-4819-158-3-201302050-00583.

15. Lan L. Study on effect of Cordyceps sinensis and artemisinin in preventing recurrence of lupus nephritis. Zhongguo Zhong xi yi jie he za zhi Zhongguo Zhongxiyi jiehe zazhi = Chinese journal of integrated traditional and Western medicine. 2002;22(3).

16. Huang XX. Clinical study on immune function regulation of artesunate in patients with lupus nephritis. Lishizhen Med. Materia Med. Res, 22: 1673-1674, 2011

17. Lin, XQ, Yuan J, Song JP, Guan YZ, Fu L, Zhou JY, Wang Q, Zhang ZJ: Experimental Study on Artemisinin Combined with Hydroxychloroquine in Treating Rats with IgA Nephropathy. Traditional Chin. Drug Res. \& Clin.Pharmacol, 27: 608-613, 2016.

18. Xia M, Liu D, Tang X, Liu Y, Liu H, Liu Y et al. Dihydroartemisinin inhibits the proliferation of IgAN mesangial cells through the mTOR signaling pathway. International Immunopharmacology. 2020;80.

\section{Figures}




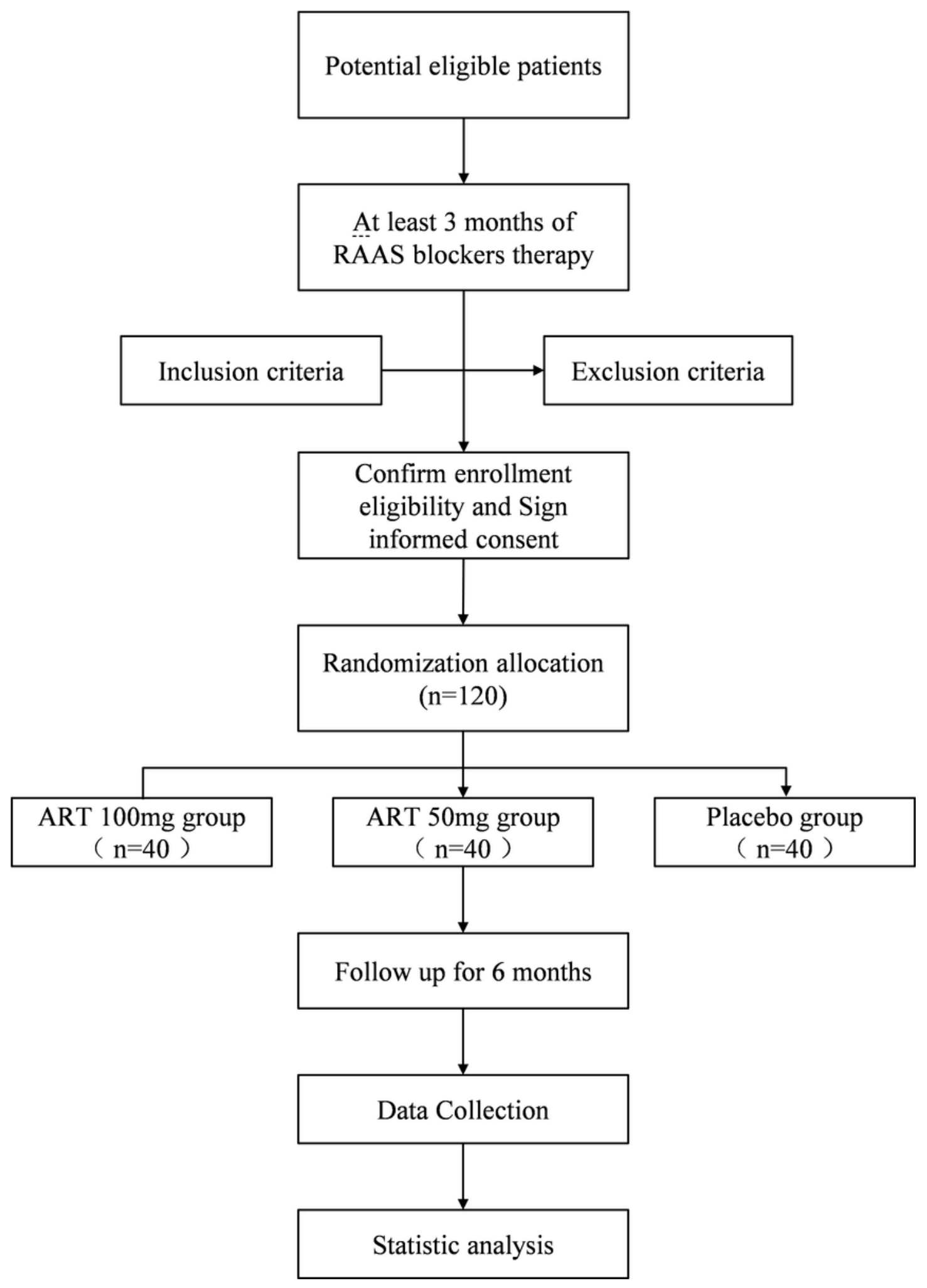

Figure 1

The flow diagram of the study progress.

\section{Supplementary Files}

This is a list of supplementary files associated with this preprint. Click to download. 
- Table1.docx

- SupplementTable1.1SPIRITChecklist.pdf 